\title{
CALEF La visibilidad del género en la práctica 29 de la educación en línea, un estudio de caso en la UAA
}

INTRODUCCIÓN

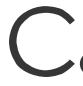

omo campo multi y transdisciplinario, los Estudios de Género (EG) han obtenido un lugar privilegiado en la sociología para explicar, desde diversas perspectivas, las diferencias entre mujeres y hombres que lo biológico no puede responder; rasgos que en ciertas situaciones son vistos como desigualdades por ser sujetos de violencia, discriminación y marginación.

Así, en esta investigación, coincidimos con la postura de Belausteguigoitia (1999), quien destaca que el "vínculo entre los estudios de género y la educación es un híbrido, multi-trans-inter-disciplinario", y que es necesario construir "conceptos de diferencia, separación, límite y frontera" (p. 9).

Los EG parten de la construcción social sobre los conceptos de mujer y hombre, del análisis de las relaciones socioculturales entre ellos y del ejercicio del poder que se vincula entre tales relaciones.

En los EG se circunscriben las teorías sobre género y el sentido de la perspectiva de género, los problemas políticos, económicos, sociales y culturales de la mujer y el hombre, pero también aquellas

1 Doctor en Ciencias Sociales y Humanidades, Universidad Autónoma de Aguascalientes. Correo electrónico: julio_montielf@live.com.mx.

2 Coordinadora Académica en la División de Estudios de Posgrado. Benemérita y Centenaria Escuela Normal del Estado de San Luis Potosí. Correo electrónico: sandrallr1@hotmail.com.mx.

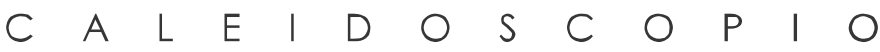


teorías que explican la homosexualidad desde el punto de vista sociocultural.

El género se comprende, en palabras de Scott (1997), como una forma de referirse a los orígenes exclusivamente sociales de las identidades subjetivas de hombres y mujeres; por tanto, el género es una categoría social impuesta sobre un cuerpo sexuado.

El género se inserta en la concepción de la diversidad sociocultural porque como tal, determina una diferencia social y cultural que en combinación con otras, constituye una característica irrefutable e inherente al ser humano (Etxeberría, 1996).

En este contexto, es valiosa la aportación de Lamas (1996), en el sentido de que son las normas culturales a partir de las instituciones (entre las que se encuentra la escuela) las que dictan a hombres y mujeres cómo comportarse en la sociedad. Así, la autora invita a un reto intelectual que lleva a repensar el valor real de lo que antes se consideraba como herencia y ahora se explica como una labor individual de interpretación de los elementos normativos y prescriptivos establecidos por la sociedad y la cultura en la que se vive un "deber ser" femenino y masculino.

En el argumento de que todos somos diferentes, la pluralidad de la cultura exige la reflexión sobre las diferencias socioculturales de los individuos para legitimar dicha diversidad sociocultural de la que todos somos parte.

Porque somos diferentes todos, porque cada una de las culturas humanas es diferente y porque la diferencia es una de las cualidades que mejor caracteriza al ser humano y precisamente esta diferencia entendida como paradigma de libertad y como elemento enriquecedor, es la que nos ayuda a organizar una percepción de la otredad que permite la construcción de una diversidad cultural no jerarquizada, basada en el conocimiento y el reconocimiento del otro (Kaplan, 1993, p. 8).

Por tanto, ser diverso socioculturalmente es reconocer las diferencias que explican las acciones del individuo sobre la organización de su vida cotidiana, de las relaciones sociales que establece y de los significados que genera a partir de ello. Así, el género representa también un principio ordenador de las relaciones sociales que no debe darse por entendido.

El género como diferencia sociocultural y elemento de la diversidad social y cultural del individuo ha servido como criterio de inclusión y exclusión en la historia de los pueblos y es referido para otorgar o impedir 
el acceso de los individuos a la participación en la toma de decisiones, al poder, a la riqueza, a la educación, etcétera.

Analizar la implicación del género en la educación significa ubicarlo como una diferencia sociocultural en un sentido de enriquecimiento y no de limitación, pues la connotación positiva de riqueza y participación hace del género una posibilidad de asumirnos en circunstancias de equidad.

Realizar investigaciones que involucren conglomerados implica un compromiso político, lo que constituye una serie de obligaciones teóricas y conceptuales entre las que se encuentran, en primer lugar, la necesidad de definir el género, el que por muchos años se consideró que únicamente implicaba aspectos físicos que aludían a lo natural o biológico, como lo han señalado autores como Butler (1997), Lamas (1997), Lauretis (1992) y otros.

Hasta hace relativamente poco tiempo estaba generalizada la idea de que ser mujer u hombre tenía fundamento en una identidad asignada desde el nacimiento. Incluso señala Lamas (1996) que algunos "pensadores ignoraban a las mujeres o esperaban que quedaran incluidas dentro de la identidad colectiva de los hombres" (p. 26). Pero nuevos estudios antropológicos han tenido como propósito identificar hasta qué punto algunos aspectos, como el género, se construyen a partir de la influencia cultural, o si se determinan por patrones biológicos. Lamas cita a Mead (1935) como una de las precursoras de "la idea revolucionaria de que los conceptos de género eran culturales y no biológicos y que podían variar ampliamente en entornos diferentes" (p. 22).

Las tendencias educativas actuales, frente a la configuración multicultural de las sociedades, muestran una inclinación hacia el respeto de las culturas y sus expresiones, y hacia una tolerancia mostrada a través de una actitud empática y conciliadora ante las diferencias.

La concepción de la educación como proceso de humanización para los individuos (Sarramona, 2008) advierte una acción dinámica del educando con otros sujetos del entorno en la que a través de los valores se proporcionan las bases para la integración social de los individuos, por tanto no admite la exclusión.

La promoción del desarrollo integral de las personas desde la educación es un deber de las instituciones que implica cambios estructurales, curriculares y en la práctica docente, con el propósito de que responda a un verdadero reconocimiento y atención de las diferencias entre las que no se puede dejar de lado al género.

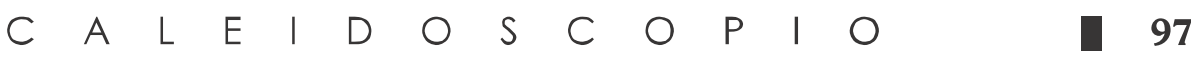


La atención homogénea de los sistemas educativos permea en la reducción de la visibilidad del género en las instituciones, pues no se reconoce en la práctica educativa la relevancia que merece la equidad, es decir, el respeto a las personas sin importar su género.

En el contexto de la educación en línea, la visibilidad del género se disipa debido a que la mediación de la educación a través de las Tecnologías de la Información y Comunicación (TIC) contribuye a que los procesos de observación obstruyan la identificación del género de manera transparente.

La modalidad educativa en línea se caracteriza por la separación en la distancia entre docente y estudiante, por lo que la interacción entre los sujetos dependerá del dominio y apropiación de las Tic, de la planificación de las actividades del curso y del estilo de enseñanza que se practique.

Los docentes forman parte importante de los procesos de enseñanza y de aprendizaje, pues son ellos quienes, como parte de su función, llevan a cabo la detección de las diferencias socioculturales de sus alumnos, y son quienes tienen como función principal atender las necesidades particulares de los estudiantes en el tránsito hacia un estado óptimo de su desarrollo.

\section{APROXIMACIÓN AL CASO}

El Diplomado de Formación de Profesores para Educación a Distancia es una opción académica ofrecida por la UAA, diseñado para la obtención de conocimientos especializados sobre estrategias educativas basadas en la aplicación de las Tecnologías de la Información y Comunicación (TIC).

El diplomado referido comenzó a funcionar en el año 2003 y contó con tres ejes de atención académica: educativo, tecnológico y comunicativo. Esta versión del diplomado se llevó a cabo en su modalidad virtual y en la actualidad también se ofrece el diplomado en modalidad semipresencial.

El diplomado está compuesto por ocho cursos que se realizan en 330 horas con un valor de 34 créditos. Los cursos son: uso de la plataforma institucional, introducción a la educación a distancia, comunicación educativa, ética en el uso de las Tic en procesos educativos, diseño de estructura para cursos, utilización de recursos tecnológicos, bases para la elaboración de materiales y taller de aplicación. 
El estudio parte de un enfoque desde el paradigma cualitativo que se orienta, según Guardián-Fernández (2007), en el interés por captar y comprender la realidad a través de la perspectiva de los actuantes, desde la percepción que ellos tienen de su propio contexto.

El método utilizado en el trabajo de investigación del que se desprende este artículo es el estudio de caso (Yin, 1994), que consiste en una investigación empírica orientada a un fenómeno contemporáneo y dentro de su contexto de la vida real, el cual es especialmente útil cuando los límites entre el fenómeno y su contexto no son claramente evidentes.

Tratar un estudio de caso, argumenta Yin (1994), conlleva una situación técnicamente distintiva, en la cual hay muchas más variables de interés que datos observacionales, y el resultado se basa en múltiples fuentes de evidencia.

El caso estudiado en la investigación referida consistió en seleccionar a un grupo de docentes con experiencia en educación en línea dentro de la institución y que tuvieron participación en el Diplomado de Formación de Profesores para la Educación a Distancia que ofrece la universidad mencionada.

\section{CRITERIOS PARA LA SELECCIÓN DE INFORMANTES}

Desde el año 2003, año en que el diplomado referido se ofreció a la comunidad de profesores y hasta el año 2013, se han formado 130 docentes de la UAA a través de siete generaciones.

La muestra es teórica y se caracteriza, según Glaser (1967), por incluir a voluntarios; se debe examinar de forma constante para decidir cuándo y dónde aproximarse, con quién conversar; con ello decimos que la muestra ha sido deliberadamente escogida y estuvo formada por 21 docentes adscritos a siete de los diez centros académicos de la Universidad Autónoma de Aguascalientes.

No se tomaron en cuenta tres centros académicos porque aún no existían durante la recolección de datos para esta investigación. El número de informantes se conformó por conveniencia y bola de nieve hasta la saturación de los temas de interés que se cumplen, en tanto no se encuentren datos adicionales para desarrollar las propiedades de las categorías (Glaser, 1967). 


\section{ESTRATEGIAS METODOLÓGICAS}

Para la recolección de datos se recurrió a la técnica de la entrevista semiestructurada, la cual define Hernández Sampieri (2010) como una conversación con la finalidad de intercambiar información entre una persona (el entrevistador) y otra (el entrevistado) u otras (entrevistados); las entrevistas cualitativas son más íntimas, flexibles y abiertas. Éstas fueron llevadas a cabo en los lugares de trabajo de los participantes en las instalaciones de la institución.

Todas las entrevistas fueron grabadas y, adicionalmente, se llevó el registro de un diario de campo. El guión de entrevista se organizó con base en categorías iniciales que, a manera de temas generales, fueron rescatados de la teoría de Lluch y Salinas (1996), Gutiez (2000) y Hofstede (2001) quienes consideraron como uno de los rasgos socioculturales esenciales el género.

El primer acercamiento se propició a partir de los datos de los participantes registrados en una base proporcionada por el área administrativa organizadora del diplomado. Para el análisis hermenéutico de la información se recurrió a la herramienta informática para el análisis cualitativo de datos ATLAS TI.

\section{DISCUSIÓN Y RESULTADOS}

Los hallazgos relacionados y obtenidos en la tesis doctoral con respecto a la importancia del género en la educación en línea, produjeron las siguientes apreciaciones que enseguida se describen.

Los docentes participantes del Diplomado de Formación de Profesores para la Educación a Distancia son personas que perciben y están conscientes de que los grupos de estudiantes que atienden son diferentes entre sí y que cada grupo concentra a sujetos con características propias que los distinguen y en las que el género constituye un rasgo que debe considerarse.

La identificación del género como diferencia sociocultural tuvo distintas apreciaciones dentro de los discursos docentes: por un lado se apreció que el género no era motivo para el ejercicio de discriminación o motivo que impidiera el objetivo educativo, sin embargo, la exploración de los datos obtenidos pudo otorgar información sobre la pertinencia de la atención del género en la educación superior en línea a través de una segunda lectura que buscamos discutir en este trabajo. 
Es importante reconocer lo que Lamas (1996) define como los "límites epistemológicos" que determinan la forma en que, por ejemplo, los docentes analizan los fenómenos relacionados con el tema, pues estos aspectos son un referente esencial de su práctica docente, así como de la manera en que interpretan las propuestas vigentes, aspecto que se relaciona con el siguiente planteamiento de la autora, que establece que "la cultura marca a los sexos con el género y el género marca la percepción de los demás" (pp.18-19).

Desde el punto de vista enriquecedor en que puede asumirse a la diversidad sociocultural - en el contexto de la educación en cualquiera de sus modalidades-, se despejan las dudas de que cualquier diferencia sociocultural pudiera ser motivo de acciones discriminatorias o de manifestaciones desiguales en su atención.

Al respecto, Lamas (1996) señala que es necesario diseñar herramientas analíticas "-conceptos, categorías, teorías- que permitan entender o, al menos visualizar, algo que antes pasaba inadvertido" (p.109). Lo que permitirá desnaturalizar los procesos que se consideran comunes.

$\mathrm{El}$ interés por retomar la perspectiva de género como una herramienta alternativa para el análisis de los procesos analizados en la investigación de la que surge el presente artículo, radica en que se coincide con la postura de Guzmán y Bolio (2010), quienes en su texto Construyendo la herramienta de género, destacan que brinda la oportunidad de analizar desde una postura diferente "los fenómenos de la vida cotidiana" (p.16).

El género como una diferencia sociocultural es admitido por los docentes como un rasgo que da coherencia a un discurso para evitar la discriminación en la educación y en el que los docentes entrevistados están convencidos que no debieran existir situaciones privilegiadas para ningún género.

Esta aseveración tiene relación con el fundamento institucional de la UAA en el que se ubica su posición humanista que subraya la formación integral de sus estudiantes con un crecimiento intelectual y personal, en equilibrio y en compromiso con su entorno. Los docentes entrevistados se remitieron a la posición institucional como un discurso que justifica el ejercicio de su práctica docente en línea.

La percepción docente sobre el género de los estudiantes está referida a ciertas actitudes, acciones y papeles de los estudiantes que, independientemente de su sexo, se relaciona con la pertenencia a la carrera en que se encuentran matriculados. 
[...] aquí participan lo mismo hombres que mujeres, aunque, bueno, sí tengo que decirlo, en la carrera de moda, mayoritariamente son mujeres, eso sí, sí es un hecho. También tenemos caballeros en la carrera, pero son pocos. Por dar un ejemplo, de 40 alumnos que entran en primer semestre, por ejemplo, hemos, eh, tenido, no sé, 37 alumnos mujeres y tres de ellos son únicamente hombres; depende mucho, hay veces que son todas mujeres, hay veces que son cinco hombres y el resto mujeres, pero, en general, mayoritariamente son mujeres, pero la participación es, en general, no tiene nada que ver, o sea, yo en lo personal no he visto ninguna, ninguna diferencia, vamos, este, pues, para mí ha sido igual ( ${ }^{*}$ Delia, 6 años de experiencia docente).

[...] Me ha tocado dar cursos en grupos donde todas son mujeres, es un reto hacerlo porque la forma en la que ven las cosas es muy distinta a como las ven los alumnos hombres, primero por la carrera y luego por la materia, pero hay muchas diferencias en cuanto a ciertas actitudes que se tienen; por ejemplo, los chavos pueden pasar por alto el contenido de materiales que uno piensa como profesor que no importa si van a hombres o mujeres, es neutral, vamos, pero cuando tienes que diseñar contenidos para un grupo total de mujeres, ellas son más sensibles a los temas que se tocan, la forma en la que ejemplificas, la manera en que realizas las actividades. Yo doy mercadotecnia y las chicas son de diseño textil o sea que tengo que poner ejemplos de modas y sí me ha costado mucho trabajo adaptar los contenidos ( ${ }^{*}$ Daniel, 7 años de experiencia docente).

Los docentes reconocen que sus grupos están formados por hombres y mujeres cuyo tratamiento y atención se considera sin distinción alguna, pero al ir profundizando sobre el tema los docentes afirman tener ciertas expectativas que van afirmando los papeles, comportamientos y actitudes que integran el género. Por ejemplo, desde su perspectiva distinguen la existencia de carreras para hombres y para mujeres; es el caso de las carreras para hombres, que están relacionadas con las ingenierías.

Respecto a lo antes referido se retoma la siguiente cita de Montesinos (2010), la autora señala que

[...] considerando la interrelación existente entre la economía, la política y la cultura, planteamos que la estructura más significativa de la sociedad es la que proviene de la división sexual del trabajo, y por tanto, la más significativa para definir la identidad tanto de hombres como mujeres (p. 14).

Lo que en relación con esta investigación implica una reflexión acerca de la forma en que la comunidad universitaria elige una carrera.

Por otra parte, se hace referencia a las expectativas de los docentes sobre el desempeño de sus estudiantes, de acuerdo a lo que socialmen- 
te se considera tradicional acerca del tipo de carrera a la que se tiene que dedicar una persona de acuerdo a su género y que deriva en trabajos "acordes al concepto socialmente aceptado de ser mujer u hombre".

Así, en el ámbito de la UAA, las carreras que tradicionalmente se han considerado aptas para mujeres son las de Diseño de Interiores, Diseño de Moda en Indumentaria y Textiles, Enfermería, Artes Escénicas, entre otras. Los informantes también incluyeron una tercera categoría que denominaron como carreras mixtas, que son las que están vinculadas con las áreas económicas y administrativas.

Respecto a las declaraciones antes descritas de los participantes en la investigación, se procedió a contrastarlas con datos estadísticos institucionales extraídos de la matrícula a nivel Licenciatura en la UAA correspondiente al semestre enero-junio de 2013, lo cual permitió reiterar la importancia de la consideración del género en la elección de una carrera. Esto se puede apreciar en las siguientes figuras que muestran el comparativo de la matrícula por género en cada una de las carreras que se ofrecen en la UAA, de al menos tres centros académicos:

Figura 1.

RELACIÓN DE HOMBRES Y MUJERES EN MATRÍCULA DE NIVEL LICENCIATURA

EN EL SEMESTRE ENE-JUN 2013. CENTRO DE CIENCIAS BÁSICAS

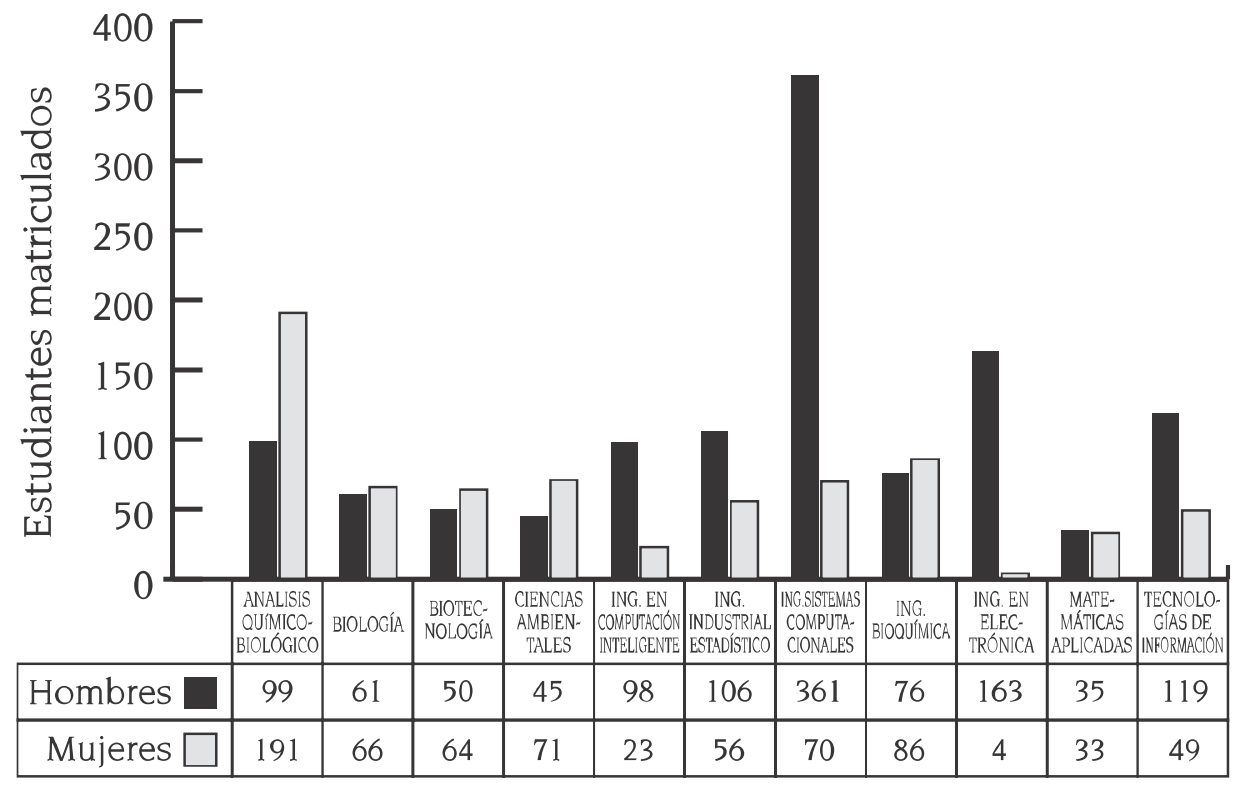

Fuente: elaboración propia a partir de información proporcionada por el Departamento de Estadística Institucional, UAA.

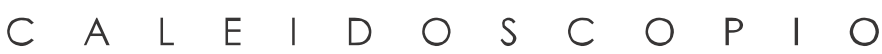


Figura 2.

RELACIÓN DE HOMBRES Y MUJERES EN MATRÍCULA DE NIVEL LICENCIATURA

EN EL SEMESTRE ENERO-JUNIO DE 2013.

CENTRO de CiENCIAS dEL Diseño y de la CONSTRUCCióN

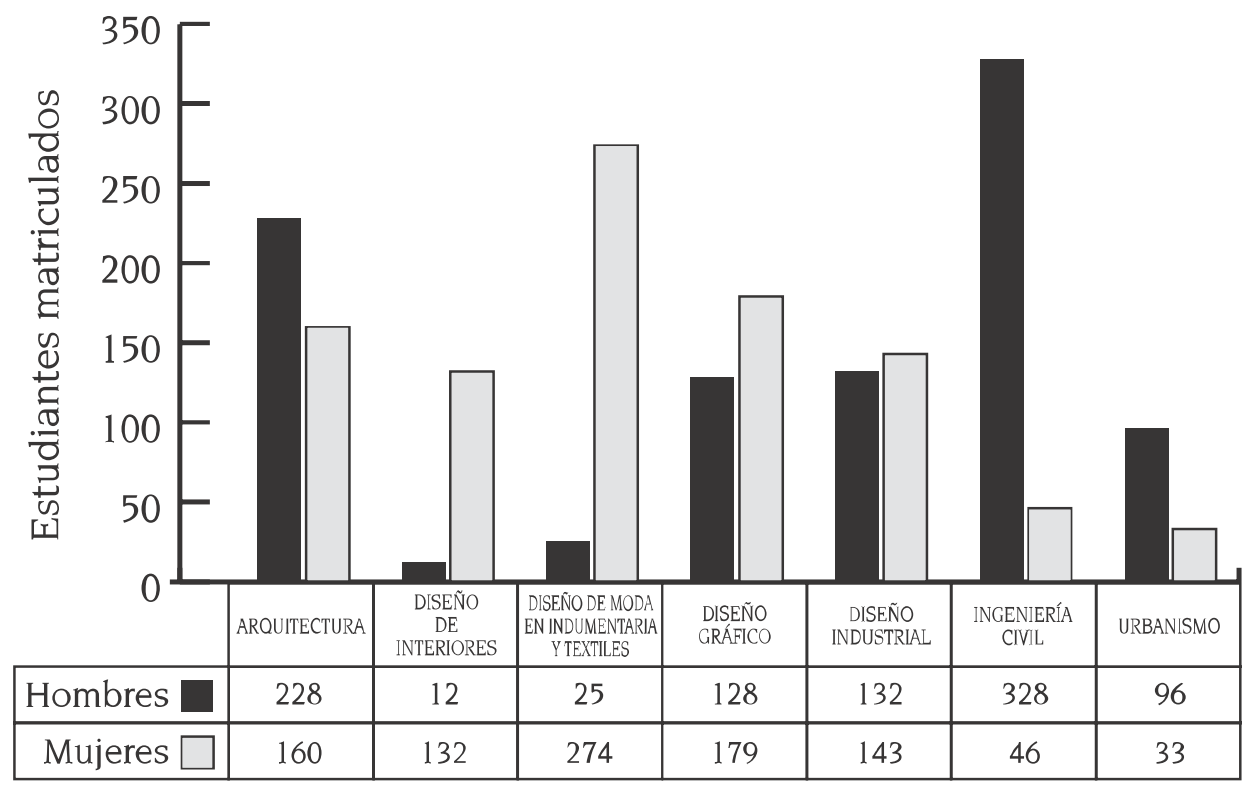

Fuente: elaboración propia a partir de información proporcionada por el Departamento de Estadística Institucional, UAA.

Hubo docentes que sostuvieron que no hay distinciones en el desempeño de los alumnos por razón de género o por su pertenencia a carreras que según creen están diseñadas para hombres o mujeres.

Los docentes entrevistados realizaron una comparación entre las formas de su práctica educativa presencial y en línea; y advirtieron que en la primera modalidad la comunicación cara a cara, verbal y no verbal, permite dar cuenta de las diferencias de los estudiantes, tanto aquellas que son visibles (rasgos físicos como el color de piel, forma de cara, partes del cuerpo, etc.) y no visibles (rasgos no observables cuya visibilidad es factible mediante la interacción entre docente y estudiante).

En la educación en línea, el sexo puede advertirse mediante mecanismos de identificación como el registro de información que contiene datos del nombre, fotografía, presentación escrita solicitada por el docente; pero el género es visible en tanto que la interacción entre docente y estudiante se realice durante la ejecución del curso. 
Figura 3.

RELACIÓN DE HOMBRES Y MUJERES EN MATRÍCULA DE NIVEL LICENCIATURA

EN EL SEMESTRE ENERO-JUNIO 2013.

CENTRO DE CIENCIAS ECONÓMICAS Y ADMINISTRATIVAS

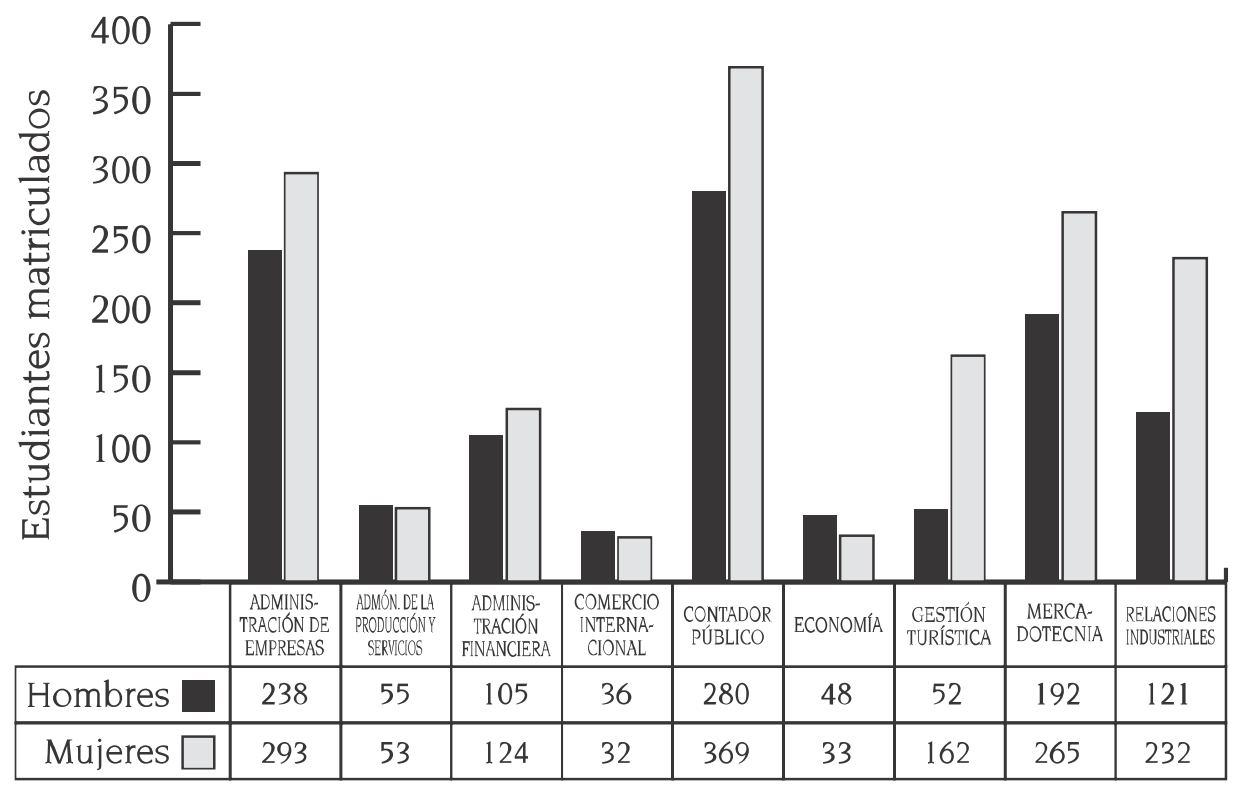

Fuente: elaboración propia a partir de información proporcionada por el Departamento de Estadística Institucional, UAA.

La interacción entre docente y estudiantes se realiza mediante herramientas tecnológicas de comunicación, dispuestas en los entornos virtuales para el aprendizaje, como pueden ser: foros, chats, correo electrónico, podcast, entre otras. El género en la educación en línea no forma parte de los rasgos socioculturales prioritarios que se reconocen por parte del docente para llevar a buen término su labor.

En la educación presencial, el docente puede obtener información de sus estudiantes con el mínimo esfuerzo de observación, incluso sin haber interactuado, y puede advertir diferencias físicas en una primera instancia, en cambio, en la educación en línea las diferencias que se advierten no son las físicas, sino las de índole sociocultural, como lo es el género, las cuales se hacen visibles de manera paulatina. 
El carácter sociocultural de los rasgos del estudiante en el nivel superior en línea lo relacionamos con un conjunto de acciones significativas que el estudiante asume y se responsabiliza de ellas (Desiato, 1996); ya sea para la construcción de las relaciones sociales o para la explicación de su realidad orientadas a la obtención de conocimiento.

En la reflexión docente de su práctica educativa en línea, la interacción y comunicación con el estudiante son aspectos primordiales que permiten la visibilidad de las diferencias socioculturales entre las que se encuentra el género. Su valoración depende de factores que el docente puede controlar a partir del grado de compromiso que asuma y que se refleja en una verdadera guía en el acompañamiento educativo.

La modalidad de la educación en línea está basada en un modelo centrado en el alumno, por lo que las diferencias socioculturales que poseen son determinantes para lograr la independencia necesaria en el medio tecnológico, motivarse internamente para concluir cada reto que se plantea y demostrar que sus diferencias socioculturales son fortalezas que el docente comprometido puede utilizar a favor del proceso de enseñanza-aprendizaje.

Pese a la naturaleza ideal de lo necesario que resulta la visibilidad y reconocimiento de la diversidad sociocultural en cualquier modalidad educativa incluida la virtual o en línea, los docentes destacaron la importancia de advertirla de forma clara en algún momento de la práctica educativa y asegurar así que se está cumpliendo con la inclusión de todos y cada uno de los integrantes del grupo.

El género, por tanto, constituye un rasgo que debe incluir más que excluir y que independientemente de ser identificado como primordial en el desarrollo de la educación en línea, su consideración puede enriquecer la base de una educación individualizada centrada en la satisfacción de las necesidades de los estudiantes que buscan lograr un objetivo educativo.

De la misma forma en que en la modalidad presencial el cuerpo físico constituye el referente primordial para identificar diferencias en proximidad con otro, en la educación en línea sólo es posible circunscribirla al producto resultante de la interacción y comunicación inducidas por el docente en una práctica deseable.

La comprensión del género en la educación en línea es fundamental para asegurar la distribución de la educación superior de forma efectiva y que mediante herramientas tecnológicas los recursos puedan funcio- 
nar adaptándose a las características sociales y culturales de cualquier ciudadano, ciudad, país o continente.

Sin embargo, la atención de este rasgo sociocultural desde la educación en línea es pertinente, pues según los docentes entrevistados, representa un factor relevante para la conformación de las relaciones sociales establecidas entre docente y estudiante, entre los mismos estudiantes y, entre estudiantes y su entorno; pero, además, es un elemento indispensable en la educación superior para determinar una práctica docente que permita la construcción de estrategias educativas a favor de la equidad y que, independientemente de que exista una apreciación de la elección de carrera por razones de sexo, requiere que el docente procure atender al objetivo educativo, más que hacer indicaciones sobre tal diferencia.

La educación en línea es un canal de acceso a la sociedad del conocimiento que puede permitir a las sociedades reducir brechas reflejadas globalmente por no contar con los recursos suficientes, flexibles y adaptables a las características socioculturales de todos los pueblos.

\section{REFERENCIAS}

Barberà, E. (2008). Aprender e-learning. Barcelona, España: Paidós.

Belausteguigoitia, M. y Mingo, A. (1999). Géneros prófugos. Feminismo y educación. México: PUEG, UNAM.

Butler, J. (1997). Variaciones sobre sexo y género: Beahuvior, Wittig y Foucault. El género: La construcción cultural de la diferencia sexual, México: Programa Universitario de Estudios de Género, UNAM.

Cabero, J. (2006). Bases pedagógicas del e-learning, recuperado el 10 de enero de 2010, de Revista de Universidad y Sociedad del Conocimiento: http://www.uoc.edu/rusc/3/1/dt/esp/cabero.pdf

Carbonell, F. (1995). Inmigración: Diversidad cultural, desigualdad social y educación. Madrid, España: Centro de Publicaciones Secretaría General Técnica, Ministerio de Educación.

Casamayor, G. C. (2008). La formación on-line: Una mirada integral sobre el e-learning, b-learning. Barcelona, España: Graó.

CPEUM. (2012). Constitución Política de los Estados Unidos Mexicanos, texto vigente.

Díaz Barriga, F., y Hernández Rojas, G. (1998). Estrategias docentes para un aprendizaje significativo, una interpretación constructivista. México: McGraw Hill.

Díaz Pintos, G. (2008). Educación y diversidad cultural. Madrid, España: Fundación Europea, Sociedad y Educación. 
Etxeberría, F. (1996). Educación y atención a la diversidad. Recuperado el 5 de septiembre de 2010, de Revista Espanola de Educacion Comparada: http://e-spacio.uned.es/fez/eserv.php?pid=bibliuned:reec-199 6-2-76A35920-9FFC-0E52-9357-01E95D48AF5FEDSID=PDF

García Martínez, A. (2008). La interculturalidad: Desafío para la educación. Madrid, España: Dykinson.

Glaser, B. S. (1967). The discovery of grounded theory: Strategies for qualitative research. New York: Aldine Publishing Company.

Guardián-Fernández, A. (2007). El paradigma cualitativo en la investigación socio -educativa. San José, Costa Rica: Colección: Investigación y Desarrollo, Educativo Regional (IDER).

Gutiez Cuevas, P. (2000). La diversidad sociocultural en el currículum de los centros educativos. Recuperado el 5 de enero de 2011, de Red de Revistas Científicas de América Latina y El Caribe, España y Portugal: http:// redalyc.uaemex.mx/pdf/567/56740104.pdf

Guzmán R., G. y Bolio M., M. (2010). Construyendo la herramienta. Perspectiva de género: cómo portar lentes nuevos. México: Universidad Iberoamericana.

Hernández de la Torre, E. (s.f.). La diversidad social y cultural como fuente de enriquecimiento y desarrollo: Aspectos conceptuales. Recuperado el 17 de septiembre de 2010, de Facultad de Ciencias de la Educación, Universidad de Sevilla: http://www.redes-cepalcala.org/inspector/DOCUMENTOS\%20Y\%20LIBROS/EDUCACION-ESPECIAL/LA\%20DIVERSIDAD\%20 COMO\%20FUENTE\%20DE\%20ENRIOUECIMIENTO.pdf

Hernández Sampieri, R. (2010). Metodología de la investigación. México: McGraw Hill.

Hofstede, G., y Hofstede, J. (2005). Cultures and organizations: software of the mind. New York: McGraw Hill.

Kaplan, A. (1993). Movimientos migratorios, movimientos culturales. Barcelona, España: Diputació de Barcelona.

Lamas, M. (Compiladora). (1996). La antropología feminista y la categoría de género. En El género: La construcción cultural de la diferencia sexual. México: PUEG, UNAM.

Lauretis, T. de L. (1992). Alicia ya no. Feminismo, semiótica, cine. Madrid: Cátedra, Universidad de Valencia, Instituto de la Mujer.

Lluch, X., y Salinas, J. (1996). Uso y abuso de la interculturalidad, Cuadernos de Pedagogía, núm. 252.

Montesinos, R. y Carrillo, R. (2010). Feminidades y masculinidades del cambio cultural de fin y de principio de siglo. En El cotidiano [en línea]. 
Sarramona, J. (2008). Teoría de la educación, reflexión y normativa pedagógica. Barcelona, España: Ariel Educación.

Scott, J. W. (1997). El género una categoría útil para el análisis histórico. En Lamas, M. (Compiladora). (1997). El género: La construcción cultural de la diferencia sexual. México: PUEG, UNAM.

Wilber, K. (2000). Una visión integral de la Psicología. México: ALAMAH.

Yin, R. K. (1994). Case study research. Design and methods. London: SAGE Publications. 